\title{
The Swedish Childhood Diabetes Study - a multivariate analysis of risk determinants for diabetes in different age groups
}

\author{
G. Dahlquist ${ }^{1}$, L. Blom ${ }^{1}$ and G. Lönnberg ${ }^{2}$ \\ ${ }^{1}$ Department of Paediatrics, Sachs' Children's Hospital, Karolinska Institute, Stockholm, and ${ }^{2}$ Department of Epidemiology and Health \\ Care Research, University of Umeå, Umeå, Sweden
}

\begin{abstract}
Summary. In a nationwide incident case-referent study stepwise univariate analysis has revealed several risk determinants for childhood diabetes mellitus. In a multivariate analysis we have determined the set of risk determinants that would independently predict childhood Type 1 (insulindependent) diabetes. Possible interactions between the risk determinants and differences in risk profiles with different ages at onset were also examined. Reported familial insulintreated and non-insulin-treated diabetes were significant risk factors in all age groups, as was also a low frequency of milk intake. The frequency of infections and a high intake of foods rich in nitrosamine tended to interact (OR 11.8, $p=0.053)$ indicating a synergistic effect. A Cox regression analysis revealed that stressful life events during the last year was the only variable that tended to affect the age at onset $(p=0.055)$. This indicated that psychological stress may rather precipitate than induce Type 1 diabetes. A short breast-feeding duration $(\mathrm{OR}=3.81)$, and an increased body height $(\mathrm{OR}=3.82)$ contributed significantly to the predictive
\end{abstract}

model in only the youngest age group (0-4 years). An increased frequency of infections in the year preceding onset $(\mathrm{OR}=2.15)$ and no vaccination against measles $(\mathrm{OR}=3.33)$ contributed significantly to the model only in the age group 5-9 years. Various nutrients had different impacts on the risk of developing Type 1 diabetes in different age groups. It is concluded that in the genetically susceptible child, risk factors which are associated with eating habits, frequency of infections, vaccination status, growth pattern and severe psychological stress affect the risk of developing diabetes independently of each other. The set of risk determinants varies with the age at onset. A high frequency of infections and a high frequency of nitrosamine-rich food intake seem to have a synergistic effect on the risk of developing diabetes in childhood.

Key words: Type 1 (insulin-dependent) diabetes mellitus, childhood, epidemiology, risk factors, case control study, multivariate analysis.
Type 1 (insulin-dependent) diabetes mellitus is thought to be the result of environmental factors which, alone or in combination, in the genetically susceptible individual, trigger an immunopathogenic process leading to Betacell destruction. The time of onset may also be determined by the need for peripheral insulin as indicated by the peak incidence occurring during puberty for both sexes [1].

The Swedish childhood diabetes study is an epidemiological project with aims at determining risk factors for the disease. It is based on the continuous registration of all new cases occurring in the 0-14 year age group [1]. An incident case-referent study covering all recent-onset diabetic children in Sweden and two age-, sex- and countymatched referent children traced with the official population register was performed during the period 1 September 1985 to 1 September 1986. The results of this study have been analysed in blocks, based on a conceptual model [2] and using standard epidemiological methods with internal standardizations. An increased risk of developing Type 1 diabetes in childhood was indicated by the following factors: 1) Reported presence of insulintreated as well as non-insulin-treated diabetes, thyroid and rheumatic disease among relatives [3]. 2) Maternal age over 40 years, low maternal education and a short duration of breast-feeding [2]. 3) A high frequency of intake of foods rich in protein, nitrosamine and nitrites/nitrates as well as complex carbohydrates and a low frequency of milk intake [4]. 4) A high frequency of infectious disease during the year preceding the onset and no vaccination against measles [5], stressed life events [6] and rapid growth (Blom L, Persson L-Å, Dahlquist G., Unpublished data).

The univariate analyses indicated that some of the risk factors were of significance only in certain age groups [2, 5]. The results of these studies have now been re-analysed 
by multivariate methods: (1) To identify which set of risk determinants will independently predict childhood diabetes when considered together. (2) To examine possible interactions between the risk determinants. (3) To analyse whether the predictive model will differ in different age groups. (4) To ascertain which of the risk determinants were important for the age at onset of the disease.

\section{Subjects and methods}

\section{Study population and methods}

The study was approved by the Ethics Committee of the Karolinska Institute and the Swedish Data Inspection Board. In the Swedish Health Care System all children aged 0-14 years with suspected diabetes are referred to paediatric departments. Since 1 July 1977 , all incident cases of diabetes are reported from the paediatric departments to a central register in Stockholm [1]. The level of detection in this register has been determined as $99 \%$ [7]. From 1 September 1985 to 31 August 1986 a total of 405 cases were registered. Twelve of the patients had refused to supply their civic number. Thus, questionnaires were sent to 393 diabetic and to 786 referent children, the latter being traced through the official Swedish population register and matched by age, sex and county. The questionnaire was returned by $339(86 \%)$ of the diabetic and by $528(67 \%)$ of the referent families. Information about vaccinations and growth charts was obtained from the children's health care centres and the school health service. There were no significant differences between those who returned and who did not return the questionnaire concerning the age, sex and county of residence of the diabetic or referent children.

\section{Statistical analysis}

Univariate and multivariate analysis was performed using the EGRET software (Epidemiological Graphics Estimation Testing. Statistical and Epidemiological Research Corporation, Seattle, Wash., USA). In the univariate analysis, the exact odds ratios and confidence limits were calculated. The tests for trend were performed by means of the Mantel-Haenszel's extension test. When three levels of exposure were used, the strata were obtained by dividing the range of the values in all individuals and designating stratum 0 as $<25$ th centile, stratum $1=25-75$ th centiles, stratum $2>75$ th centiles. Length was defined as a risk factor when the standard deviation (SD) score (the individual length expressed as a multiplicr of the SD of a reference population) by age and sex was $>0.1$. The mean of growth measurements recorded 5-1 years before onset of the disease was then used. As in the univariate tests, the ratios between a matched and an unmatched analyses were close to one the matching was dissolved [8] in order to improve the precision of the analysis.

In the multivariate analysis only dichotomous variables were used. The dichotomous variables for milk and solid food protein, nitrosamine and carbohydrate used stratum $1+2$ as the exposed level. The logistic regression models were fitted by means of maximum iikelihood estimations. Whereas the general form of a regression analysis is $Y=\beta_{0}+\beta_{1} X_{1}+\beta_{2} X_{2} \ldots$ where $Y$ is predicted by a set of variables and their coefficients, the logistic regression analysis models the logarithm of the odds of Y i. e. $\ln \frac{y}{y-1}$ (also called the logit) and the regression model then shows the dependence of an expected proportion or probability of $Y$ on $x$. In the present study, modelling the probability of being a diabetic, the assumption was that of a multiplicative association to the different exposures; thus, in each exposure, the term $\mathrm{e}^{\beta_{i}} \mathrm{x}_{i}$ is used. In the analysis the $\log$ values are used to obtain a linear model. Then the relative risk (expressed as an odds ratio) for diabetes when children were exposed to the risk factor is exp

Table 1. Univariate statistics on risk factors for Type 1 (insulin-dependent) diabetes in childhood

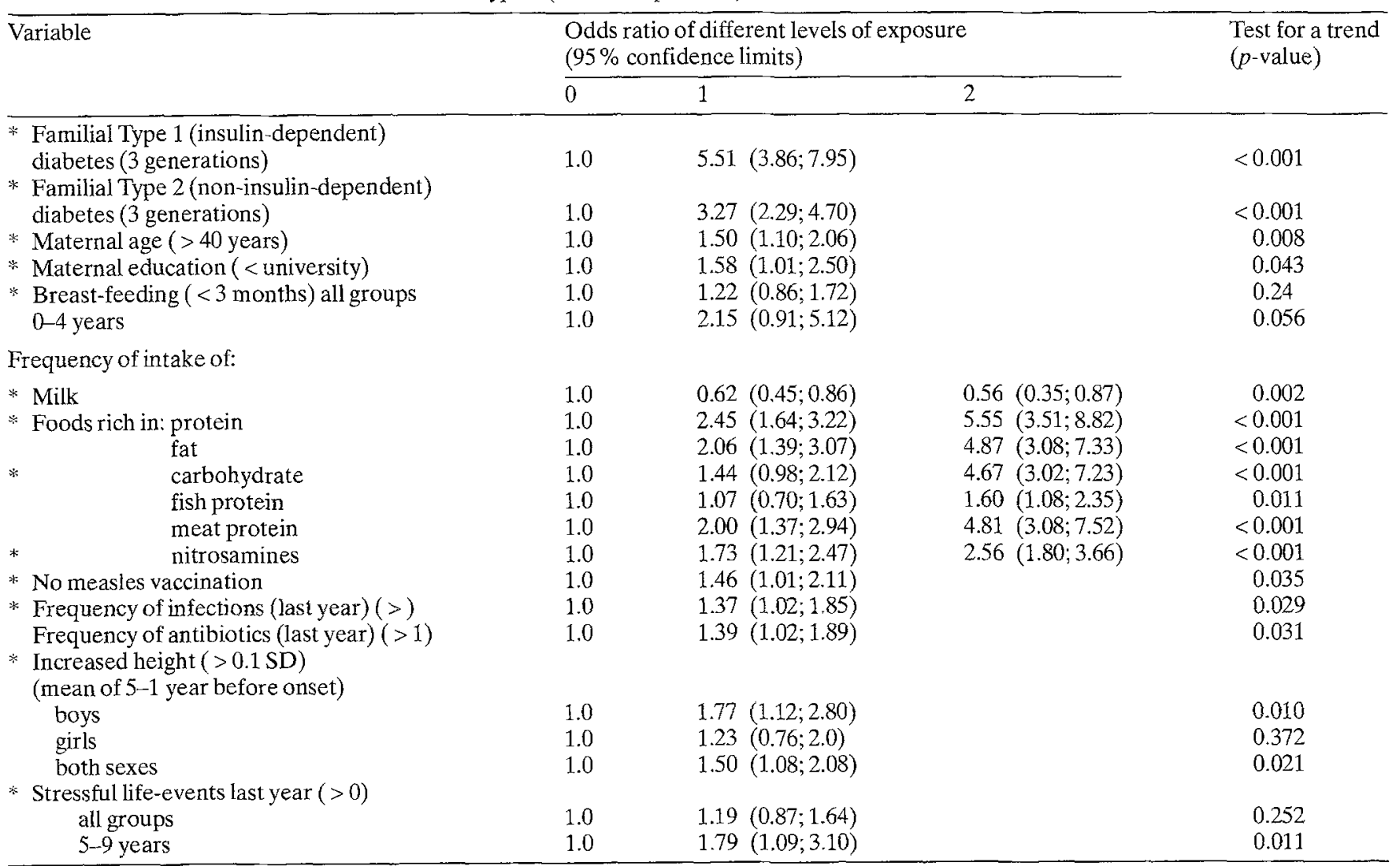

* Denotes the variables included in the first step of the logistic regression analysis of the risk determinants of diabetes shown in Table 4 
Table 2. Logistic regression model including all identified significant risk determinants for Type 1 (insulin-dependent) diabetes in childhood in age group 0-14 years

\begin{tabular}{|c|c|c|}
\hline Variables & $\begin{array}{l}\text { Odds ratio } \\
\text { (95\% confi- } \\
\text { dence limits) }\end{array}$ & $p$-value \\
\hline $\begin{array}{l}\text { Familial Type } 1 \text { (insulin-dependent) } \\
\text { diabetes } \\
\text { Familial Type } 2 \text { (non-insulin-dependent) } \\
\text { diabetes }\end{array}$ & $\begin{array}{l}5.09(3.11 ; 8.31) \\
2.79(1.70 ; 4.58)\end{array}$ & $<0.001$ \\
\hline Maternal age ( $\geq 40$ years) & $1.24(0.78 ; 1.96)$ & 0.357 \\
\hline Maternal education ( < university) & $0.94(0.52 ; 1.71)$ & 0.846 \\
\hline Breast-feeding ( $<3$ months) & $1.06(0.66 ; 1.70)$ & 0.804 \\
\hline $\begin{array}{l}\text { Frequency of intake of: } \\
\text { milk ( }<25 \text { th centile })\end{array}$ & $2.03(1.34 ; 3.07)$ & $<0.001$ \\
\hline $\begin{array}{l}\text { Foods rich in: } \\
\text { protein }(>25 \text { th centile }) \\
\text { carbohydrate }(>25 \text { th centile }) \\
\text { nitrosamines }(>25 \text { th centile })\end{array}$ & $\begin{array}{l}2.63(1.48 ; 4.68) \\
1.86(1.14 ; 3.04) \\
1.52(0.99 ; 2.32)\end{array}$ & $\begin{array}{l}0.001 \\
0.013 \\
0.056\end{array}$ \\
\hline No measles vaccination & $1.54(0.93 ; 2.54)$ & 0.093 \\
\hline Frequency of infections last year $(>1)$ & $1.49(0.97 ; 2.27)$ & 0.067 \\
\hline $\begin{array}{l}\text { Increased height }(>0.1 \mathrm{SD}) \\
\text { (Mean of } 5-1 \text { years before onset) }\end{array}$ & $1.37(0.89 ; 2.11)$ & 0.147 \\
\hline Stressful life-events last year $(>0)$ & $1.06(0.67 ; 1.67)$ & 0.798 \\
\hline
\end{tabular}

Deviance on $513 d f=571.26$

Likelihood ratio statistics on all included variables $14 d f=159.32$ $p<0.001$

Table 3. Cox regression on risk determinants for age at onset of childhood diabetes

\begin{tabular}{llr}
\hline Variables & Hazard ratio & $p$-value \\
\hline $\begin{array}{l}\text { Familial Type 1 (insulin-dependent) } \\
\text { diabetes }\end{array}$ & 1.05 & 0.735 \\
Familial Type 2 (non-insulin-dependent) & 0.91 & 0.552 \\
diabetes & & \\
Maternal age ( > 40 years) & 0.45 & $<0.001$ \\
Maternal education ( < university) & 1.24 & 0.338 \\
Breast-feeding (<3 months) & 0.86 & 0.343 \\
Frequency of intake of & & \\
$\quad$ milk ( <25th centile) & 0.93 & 0.620 \\
Foods rich in & & \\
$\quad$ protein ( $>25$ th centile) & 0.70 & 0.161 \\
$\quad$ carbohydrate ( $>25$ th centile) & 1.00 & 0.997 \\
$\quad$ nitrosamine ( $>25$ th centile) & 1.01 & 0.946 \\
No measles vaccination & 0.83 & 0.261 \\
Frequency of infections last year $(>1)$ & 0.91 & 0.507 \\
Increased height $(>0.1$ SD) & 1.07 & 0.658 \\
Stressful life-events last year $(>0)$ & 1.35 & 0.055 \\
\hline
\end{tabular}

Deviance 1916.42

Likelihood ratio statistics on all included variables $13 d f=33.28$ $p=0.002$

$\beta$. The likelihood ratio statistic measures the significance of the model. The null hypothesis is that the coefficients for all the parameters are simultaneously zero. The deviance of the model is the likelihood ratio statistics between the fitted model and the completely saturated model and can be used as a measure of the goodness of fit. The independent significance of an estimated coefficient of a variable in the model was tested using the Wald test statistic which, in general, will be close to the value of a corresponding likelihood ratio statistic, computed as the difference between the deviances of the models with and without the respective term. Ninety-five percent confidence limits were calculated using the iterative method. To analyse the determinants of age at onset for the cases, Cox's proportional hazards regression was used. This model is normally used to analyse follow-up data as it models the failure time. Though the present study is crosssectional in nature the diabetic cases can be regarded as followed from birth to the time at onset and thus the time at onset of diabetes can be modelled as a function of the recorded exposures. All the statistical methods used have been described previously [9].

\section{Results}

\section{Univariate statistics}

A summary of the results of the univariate statistics are given in Table 1. Breast-feeding and stressful life-events were associated with a significant risk only in certain age groups. A high intake of some of the different nutrients are related and were therefore standardized for each other. Thus, when the frequency of fat intake was standardized for protein intake the trend in odds ratio by exposure level was no longer statistically significant, whereas the frequency of protein intake still showed a significant trend in the odds ratio when standardized for fat. Nutrients rich in protein and nitrosamine nutrients were still associated with a significant trend when standardized for each other. Since fish protein and meat protein are both associated with an increase in trend these nutrients were combined. The frequency of infections was closely related to the frequency of intake of antibiotics, and when standardized for each other neither trend remained significant. In the multivariate statistics we chose to use frequency of infections but not the use of antibiotics as a risk factor. The girls and boys were analysed separately for height. For boys but not girls, the odds ratio remained statistically significant.

\section{Multivariate analyses}

Logistic regression of risk determinants in all age groups and interactions. The risk of being a diabetic child was first modelled using the variables indicated by an asterisk in Table 1. The "fit" model is shown in Table 2. In this model reported familial diabetes, both insulin-treated and noninsulin-treated, and specific eating habits were the strongest independent risk determinants. Maternal education and age were no longer significant indicating that these variables affect the risk only indirectly through some of the other included risk determinants.

To analyse possible interactions between the terms of this fit the significant terms of the model were combined in pairs. A clear tendency to interaction was found for "the frequency of infections in the last year" $\times$ "a high frequency of intake of nutrients rich in nitrosamine" $(\mathrm{OR}=11.79, p=0.053)$ thus indicating a synergistic effect of the two variables.

\section{Cox regression of determinants for age at onset of the diabetic children}

The model included all risk determinants indicated by an asterisk in Table 1 . The outcome variable was age at diagnosis in months. Table 3 shows that the only variable (ex- 
Table 4. Logistic regression analysis of the risk determinants for diabetes with onset at 0-4, 5-9 and 10-14 years of age, respectively. In the first step all variables denoted by * in Table 1 were included. In the second step, for each age group only risk determinants yielding a $p$-value $<0.20$ were included

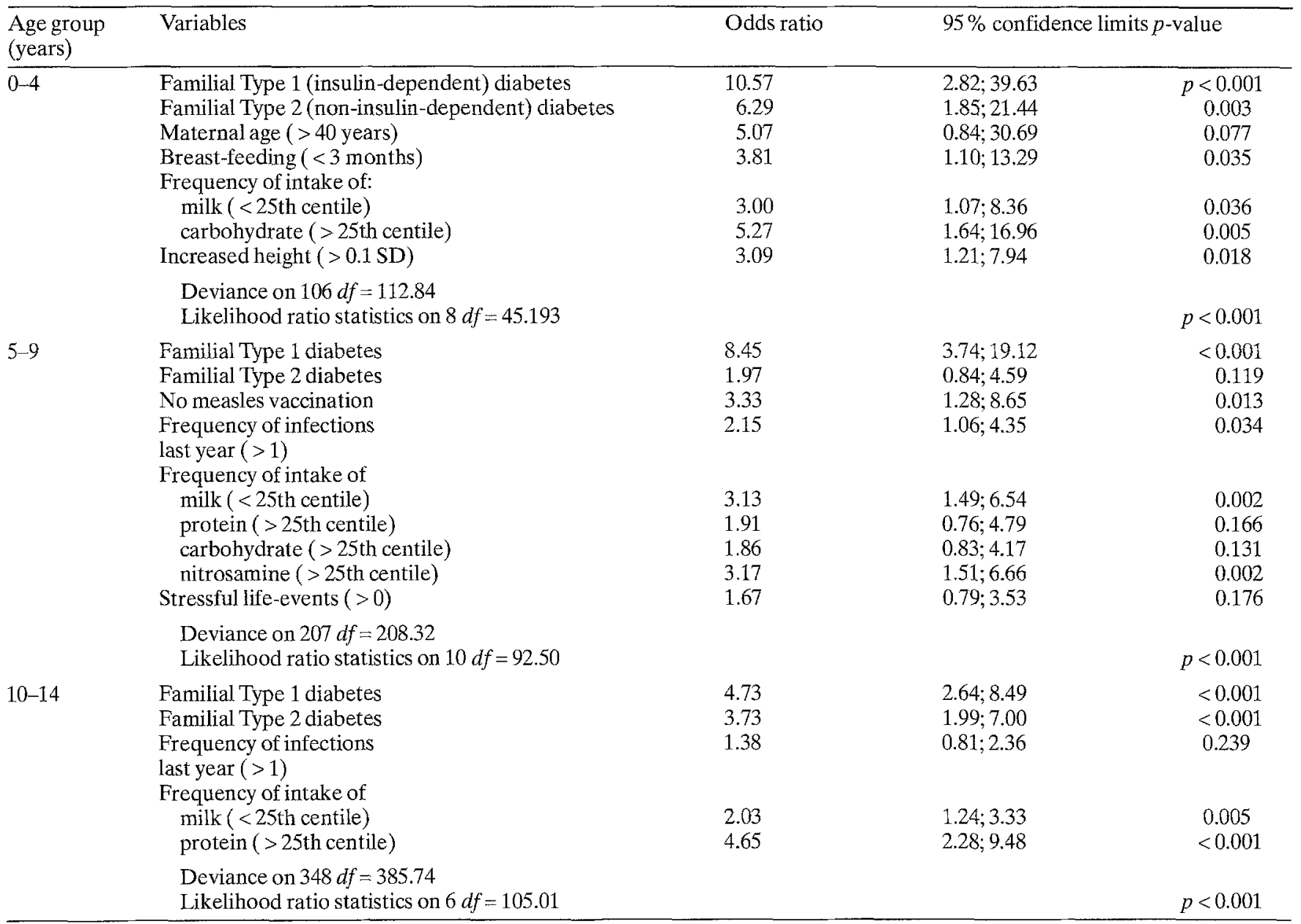

cept the obvious association with maternal age) which tended to determine the age at onset was stressful lifeevents occurring during the year preceding diagnosis.

\section{Modelling the risk of developing diabetes in different age groups}

The variables of the model given in Table 2 were modelled for children $0-4,5-9$ and $10-14$ years, respectively, and terms yielding a $p$-value $>0.20$ were excluded to yield the new models shown in Table 4. Different risk profiles emerged in the different age groups with a breast-feeding duration of less than 3 months and increased height being significant only among the youngest children. The protective effect of measles vaccination and the increase in risk with an increased number of infectious diseases were significant only among children in the 5-9 years age group. Except for a low frequency of intake of milk, that added to the regression in all age groups, the various nutrients had different impact on the risk model in the three age groups. No specific patterns were, however, found when analysing the data by sex.

\section{Discussion}

The analyses primarily performed in the Swedish casecontrol study, concentrating on non-genetic risk determinants for childhood-onset Type 1 diabetes, was based on a conceptual model of multifactorial causation [2] and carried out in steps according to this model. The analyses revealed a set of thirteen significant risk determinants the independence of some of which could be studied using standard stratification strategies. To study the effect on each of these thirteen risk determinants unconfounded by all the others, however, a multivariate modelling of the data was necessary simply because further stratification would yield too small strata. The multivariate analysis is also useful when looking for possible interactions between risk determinants e.g. synergistic effects that may be of a considerable biological interest. Therefore, even if a stratified analysis yields more straightforward interpretations and provided each multivariate model is based on a relevant biological hypothesis, this kind of analysis may provide important new knowledge.

When all thirteen risk determinants for childhood diabetes were included in a logistic regression model social 
risk determinants, such as maternal age and education disappeared whereas risk factors, related to eating habits, remained significant, indicating that the latter maydependon the former. A clear tendency towards a positive interaction between the total frequency of infectious diseases during the year preceding the onset of diabetes and a high frequency of intake of nitrosamine-rich compounds was revealed, which meant a more than ten-fold increase in risk. A high frequency of infections may be part of the process that leads to diabetes by two different mechanisms, firstly by non-specific release of interleukin 1 which has proved to be of pathogenic importance for Beta-cell destruction [10], secondly by an increase in the need for peripheral insulin. There are several indications that nitrosamine compounds are diabetogenic in experimental animals $[11,12]$ and that the pathogenic action takes place at Beta-cell level. The positive interaction between these two risk determinants indicates that a Beta-cell toxic action of a high frequency of nitrosamine-rich food intake may be enhanced by a simultaneous exposure to infectious diseases.

Quite different risk profiles were found in children of different ages. In the youngest age group a short breastfeeding period and a high growth rate were important risk determinants not seen in the older age groups. A high growth rate may be a stress factor for the Beta cell since it increases the demand for insulin production probably via an increase in peripheral insulin resistance induced by high levels of growth hormone [13]. A short duration of breastfeeding has repeatedly been shown to be associated with an increased risk of Type 1 diabetes [13-15]. The mechanism for this increased risk may be either a lack of immunological protection by the mother's milk or be dependent on the early introduction of cow's milk formula which may trigger a pathological immune response. The latter hypothesis accords with the findings of increased levels of IgA-antibodies to both cow's milk and betalactoglobulin, especially in young recent-onset diabetic children [16]. Our finding of a lower frequency of milk intake among diabetic children may indeed be in accordance with the idea of an early introduction of cow's milk proteins leading not only to cow's milk antibodies but also perhaps to clinical cow's milk intolerance which, in turn would lead to a decrease in milk intake after the first years of life.

Among children 5-9 years of age the frequency of infections and vaccination against measles were important independent risk determinants. Vaccination against measles was introduced in Sweden as a voluntary vaccination in 1971, but since 1982 it has been part of the general vaccination programme, therefore, among the youngest children almost all cases and referent children have had this vaccination. This may explain why the protective effect will emerge only among older children. Measles is a serious infectious disease which leads to a series of immunological reactions and also probably to an intense inflammatory response with high levels of interleukin 1 release. Thus, the protective effect of measles vaccination may be both specific and non-specific.

In the univariate analysis a stressful life-event i.e. a separation within the family such as a divorce or the death of a family member was a risk factor only in the 5-9 year age group. In the multivariate analysis, however, the stat- istical significance of this factor disappeared, even in this age group. On the other hand, among the risk determinants for the age of disease onset, a stressful life-event was the only one that tended to have a significant effect. The interpretation may be that stressful life-events may have an effect on the time of onset of diabetes, perhaps by increasing the levels of catecholamines which in turn increase the peripheral insulin requirement [17]. However, when compared to other risk factors of pathogenic importance for Beta-cell destruction, this risk factor may be of little significance. It should be noted that the lack of significant associations of the other risk determinants to the time of disease onset may be due to small numbers as well as the cross-sectional study design and only a prospective study may more distinctly exclude such associations.

It is interesting to note that the frequency of intake of foods rich in carbohydrate, protein and nitrosamine varies as risk factors in different age groups, probably because of differences in eating habits between the age groups. A low frequency of milk intake appeared as a strong risk factor in all age groups. The possibility of a connection between these findings and the early introduction of cow's milk proteins leading to an increased susceptibility to cow's milk protein intolerance must be further examined.

In conclusion, this study has shown that risk factors associated with an increased growth and with lifestyle habits such as duration of breast-feeding and intake of different foods, as well as the total load of infectious diseases, are important and independent risk factors for the development of Type 1 diabetes in childhood. The risk profiles seem to differ significantly in different age groups. Stressful life-events may be of importance for the time of onset of the disease.

The different risk profiles in different age groups and the independence of some of the identified risk determinants as well as the indicated synergism support the idea of a complex mode of disease causation. In the genetically susceptible individual different triggers may induce and/or accelerate the immunopathogenic process in the Beta cell; whereas other risk factors acting perhaps by increasing the insulin need may precipitate the disease occurrence.

The associations with risk determinants related to lifestyle habits should be further studied in different countries. This may provide a basis for primary intervention strategies at the population level that may effectively stop the increasing incidence of childhood Type 1 diabetes now reported from many countries in the world.

Acknowledgements. The Swedish Childhood Diabetes Study is supported by grants from the Swedish Medical Research Council, project No. B87-27X-07531, the Karolinska Institute, Nordisk Insulin Foundation, the Swedish Diabetes Association, and the Swedish Diabetes Foundation. We also thank all the paediatricians and diabetes nurses throughout Sweden for diligently sending in the registration forms to the Swedish Childhood Diabetes Register.

\section{References}

1. Dahlquist G, Blom L, Holmgren G, Hägglöf B, Larsson $\mathrm{X}$, Wall $S(1985)$ The epidemiology of diabetes in Swedish children 0 14 years - A six year prospective study. Diabetologia 28: 802-808 
2. Blom L, Dahlquist G, Nyström L, Sandström A, Wall S (1989) The Swedish childhood diabetes study - Social and perinatal determinants for diabetes in childhood. Diabetologia 32: 7-13

3. Dahlquist G, Blom L, Tuvemo T, Nyström L, Sandström A, Wall S (1989) The Swedish childhood diabetes study - Results from a nine year case register and a one year case-referent study indicating that Type 1 (insulin-dependent) diabetes mellitus is associated with both Type 2 (non-insulin-dependent) diabetes mellitus and autoimmune disorders. Diabetologia 32:2-6

4. Dahlquist G, Blom L, Persson L- $\AA$, Sandström A, Wall S (1990) Dietary factors and the risk of developing insulin dependent diabetes in childhood. Br Med J 300: 1302-1306

5. Blom L, Nyström L, Dahlquist G (1991) The Swedish childhood diabetes study: vaccinations and infections as risk determinants for diabetes in childhood. Diabetologia 34:176-181

6. Hägglöf B, Blom L, Lönnberg G, Sahlin B, Dahlquist G (1991) The Swedish childhood diabetes study: indications of severe psychological stress as a risk factor for Type 1 (insulin-dependent) diabetes in childhood. Diabetologia 34:579-583

7. Nyström L, Dahlquist G, Rewers M, Wall S (1990) The Swedish childhood diabetes study. An analysis of the temporal variation in diabetes incidence 1978-1987. Int J Epidemiol 19: 141-146

8. Miettinen O (1972) Components of the crude risk ratio. Am J Epidemiol 96: 168-172

9. Breslow NE, Day NE (1980) Statistical methods in cancer research. Vol I - The analysis of case-control studies. Lyon, International Agency for Research on Cancer (IARC Scientific Publications No 32)

10. Nerup J, Mandrup-Poulsen T, Molvig J, Helqvist S, Wogensen L, Egeberg $\mathfrak{J}$ (1988) Mechanisms of pancreatic $\beta$-cell destruction in Type 1 diabetes. Diab Care 11: 16-23

11. Gunnarsson R, Berne C, Hellerström C (1974) Cytotoxic effects of streptozotocin and $\mathrm{N}$-nitrosometylurea on the pancreatic B- cells with special regard to the role of nicotinamide - adenine dinucleotide. Biochem J 140: 487-494

12. Helgason T, Ewen SW, Ross JS, Stowers JM (1982) Diabetes produced in mice by smoked/cured mutton. Lancet II: 1017-1022

13. Rizza RR, Mandarino LJ, Gerich JE (1982) Effects of growth hormone on insulin action in man. Mechanisms of insulin resistance, impaired suppression of glucose prediction and impaired stimulation of glucose utilization. Diabetes 31: 663-669

14. Borch-Johnsen K, Joner G, Mandrup-Poulsen Tet al. (1984) Relation between breast-feeding and incidence rates of insulin-dependent diabetes mellitus. A hypothesis. Lancet II: 1083-1086

15. Mayer EJ, Hamman RF, Gay EC, Lezotte DC, Savitz DA, Klingensmith GJ (1988) Reduced risk of Type 1 (insulin-dependent) diabetes mellitus among breast-fed children. Diabetes 37: 16251631

16. Savilahti E, Åkerblom HK, Tainio V-M, Koskimies S (1988) Children with newly diagnosed insulin dependent diabetes mellitus have increased levels of cow's milk antibodies. Diab Research $7: 137-140$

17. Lager I, Attvall S, Eriksson BM, v Schenk H, Schmit U (1986) Studies on the insulin-antagonistic effect of catecholamines in normal man. Diabetologia 29: 409-416

Received: 17 April 1991

and in revised form: 12 July 1991

Dr. G. Dahlquist

Department of Paediatrics

Karolinska Institute

Sachs' Children's Hospital

S-11895 Stockholm

Sweden

\section{Erratum}

Diabetologia, Volume 34, Supplement 2, August 1991, pp. A49, Abstract 192

L. T. Diemel et al.: Neuropeptide status in experimental diabetic neuropathy

Line 11 of the main text of this abstract should read "... rats (respectively $0.14 \pm 0.02$ and $4.42 \pm 0.44$ ) compared to" and line 12 of the main text should read " . . controls $(0.23 \pm 0.02$ and $9.06 \pm 0.65$; both $p<0.05$ ). 